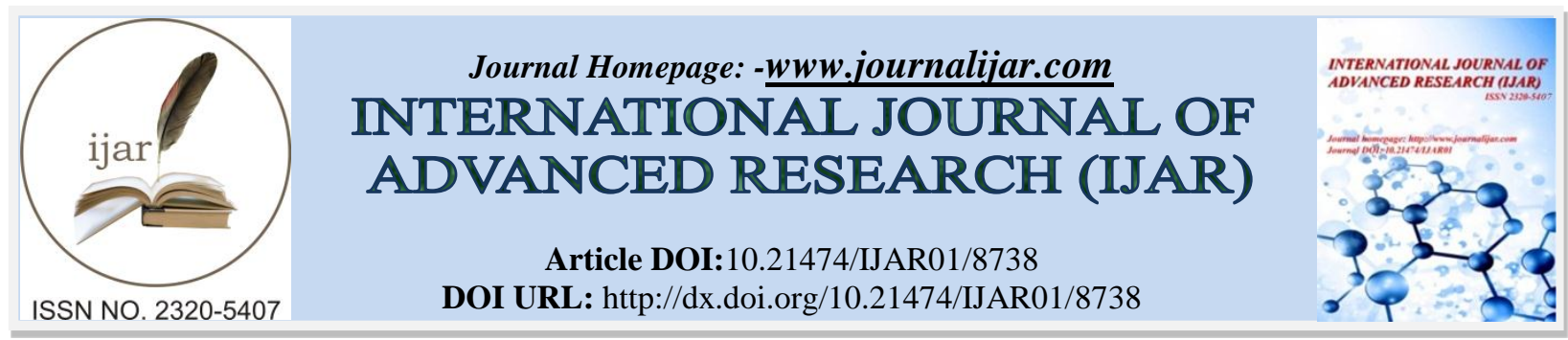

RESEARCH ARTICLE

\title{
PREVALENCE OF POSTPARTUM DEPRESSION AMONG PRIMIGRAVIDAE AND MULTIGRAVIDAE WOMEN IN MUMBAI.
}

\section{Dr. Pranita Ganjave ${ }^{1}$ and Mansi Patel ${ }^{2}$.}

1. Assistant professor, School of Physiotherapy, D.Y.Patil University.

2. Final year Student, School of Physiotherapy, D.Y.Patil University.

\section{Manuscript Info}

Manuscript History

Received: 19 January 2019

Final Accepted: 21 February 2019

Published: March 2019

Key words:-

Risk, EDPS ,Multigravidae, Primigravidae, Depression,Postnatal

\section{Abstract}

Aim of the study was to find out prevalence of postpartum depression in primigravidae \& multigravidae women in mumbai.

Method: A survey based cross sectional study was done .A total of 100 subjects(50 multigravidae \& 50 primigravidae) were recruited for the study.A self made questionnaire \& Edinburgh postnatal depression scale were used for assessment.Multigravidae \& primigravidae women more than 1 week postpartum were selected for the study.Subjects with previous /existing history of psychiatric illness and associated depression were excluded from the study.

Results: Out of 100 women, $36 \%$ women showed presence of postnatal depression. In which $24 \%$ multigravida \& $20 \%$ primigravida women had EDPS score falling in high risk category whereas $16 \%$ multigravidae \& $12 \%$ Primigravidae women had EDPS score falling in borderline category.Between group comparison showed no significance difference between Primigravidae and Multigravidae women EDPS score $(\mathrm{p}>0.05)$.

Conclusion: From the above study we conclude that both the primigravidae as well as multrigravidae women showed the presence of post natal depression.

Copy Right, IJAR, 2019,. All rights reserved.

\section{Introduction:-}

Childbirth brings many biological, psychological and social changes in a woman's life. These changes can contribute to personal growth and happiness, but may also predispose women to emotional distress.[1] Life with the new born can be very thrilling and rewarding but at the same time, it can be very hard and strenuous for some. This beautiful moment of having a new born brings about many physical, hormonal and emotional changes in the women during childbirth. These changes, collectively with other compounding factors, may lead to a feeling of sadness, anxiety, scare and confusion among many mothers. Such feelings make it extremely difficult for the mother to take care of herself or to tend to the needs of the new born. This in turn puts a lot of strain on the family relationships. For most mothers these feelings are temporary and disappear as quickly as they appear but for some unfortunate mothers, it not only remains but develops into a serious yet common disorder known as postpartum depression (PPD). 
Postpartum depression is a type of mood disorder associated with the child birth in which there is presence of depressive symptoms in postpartum period. Negative or ambivalent feelings towards the child or doubts about motherhood quality are generally reported by women with postpartum depression. If unrecognised and left untreated, can develop into more severe condition called postpartum psychosis, which can lead to foeticide or maternal suicide. [2]

The DSM-IV-TR (diagnosis and statistical manual of mental disorders) recognises postpartum depression as a major depressive disorder with postpartum onset and indicates depressive symptoms begins within 4 weeks postpartum. ICD-10 recognises postpartum depression as a mild mental and behavioural disorder commencing within 6 weeks of delivery[3] [4].

Depressed mothers express more negative emotions (such as sadness, anxiety, nervousness, aggression), perceive more difficult relationship with their family. They have little verbal communication with their infants. There is decreased utilisation of preventive services for infants such as vaccination. Additionally, infants of depressed mothers shows impaired maternal-child interactions, lower cognitive development, more behavioural problems and higher risk of psychiatric disorders during adolescent years.[5] It has been observed that quality of life and daily functioning of mothers and infants were negatively impacted by postpartum depression.[6][7]Several caregiving activities also appear to be compromised by postpartum depression including feeding practices, most especially breastfeeding, sleep routines and well child visits, vaccinations and safety practices.[8]Women who have postnatal depression are significantly more likely to experience future episodes of depression Anxiety and depressionis common in life, it is also common among Primigravida and multigravida mothers during labor and delivery.[9] Mothers suffers from severe mental stress during their labor, delivery and hospitalization. Providing psychological support is one of the most important need of the time in order to prevent the mothers from this mental depressed state. [1] Post-partum depression is generally considered a biomedical psychiatric category, attributed to genetic, hormonal and biological factors and treated with antidepressant medication. [10]

Postpartum depression not only affects the mental health and physical abilities of mothers but also on long run basis puts a detrimental effect on mother-infant relationship contributing towards child's cognitive and emotional lacking/deprivation. Depression is one of the most commonly experienced features of post-partum depression and women particularly in our society often remains undiagnosed and untreated.

In most developed nations problem of Postpartum Depression is often overlooked, also many mothers are unaware about postpartum depression, which explains as to why there has been paucity of research on postpartum depression in developing countries. It is an area to be concerned that there is lack of knowledge and awareness regarding postpartum depression in our society and the diagnosed cases are not provided any medical assistance.

There are very few studies carried out in India to find out the prevalence of post partum depression and they all show grossly varied results. This may lead to over diagnosis or under diagnosis of the condition. So study conducted to find out the prevalence of postpartum depression and the factors associated with post natal depression.

\section{Materials and Methodology:-}

A self made questionnaire \& Edinburgh Postnatal Depression rating scale ( EDPS) were used for assessment. It was a survey based cross sectional study in which 100 [50 Multigravidae \& 50 Primigravidae] were interviewed. Inclusion criteria for the study was multigravidae and primigravidae women more than 1 week postpartum. Whereas, subject with previous /existing history of psychiatric illness and associated depression, Multigravidae women less than 1 week postpartum, Primigravidae women less than 1 week postpartum were excluded from the study.

Edinburgh Postnatal Depression rating scale(EDPS)[11] Edinburgh Postnatal Depression Scale (EPDS) is a 10-item self rating questionnaire that was developed to identify women who have postpartum depression. Items of the scale correspond to various clinical depression symptoms, such as guilt feeling, sleep disturbance, low energy, anhedonia, and suicidal ideation. Overall assessment is done by total score, which is determined by adding together the scores for each of the 10 items. Higher scores indicate more depressive symptoms. The cut- off point was 13 or above for high risk and 10 or above for borderline EDPS score. Sensitivity \& specificity of EDPS scale was found to be $80 \%$ $\& 87 \%$ respectively. [12] 
Procedure - Institutional Ethic committee approval was taken before the starting of the study. Aim of conducting survey \& purpose of conducting research were explained to the patient. Consent of patient was taken with assurity that their identity will be kept anonymous \& data collected will be used only for academic research purpose. Self made questionnaire containing Socio-Demographic details, Obstetric, physical, cultural factors was validated.multigravidae and primigravidae women were interviewed for the information. Edinburgh Postnatal Depression rating scale was used to assess postnatal depression.

Statistical Test- Statistical analysis was done using SPSS 16. Descriptive statistics was used. Normality of data was determined by Shapiro-Wilk Test. Since the data was not normally distributed Mann-Whitney U test was used for between group comparison.

Graph 1:- Factors Affecting Postpartum Depressionin Multigravidae and Primigravidae Women

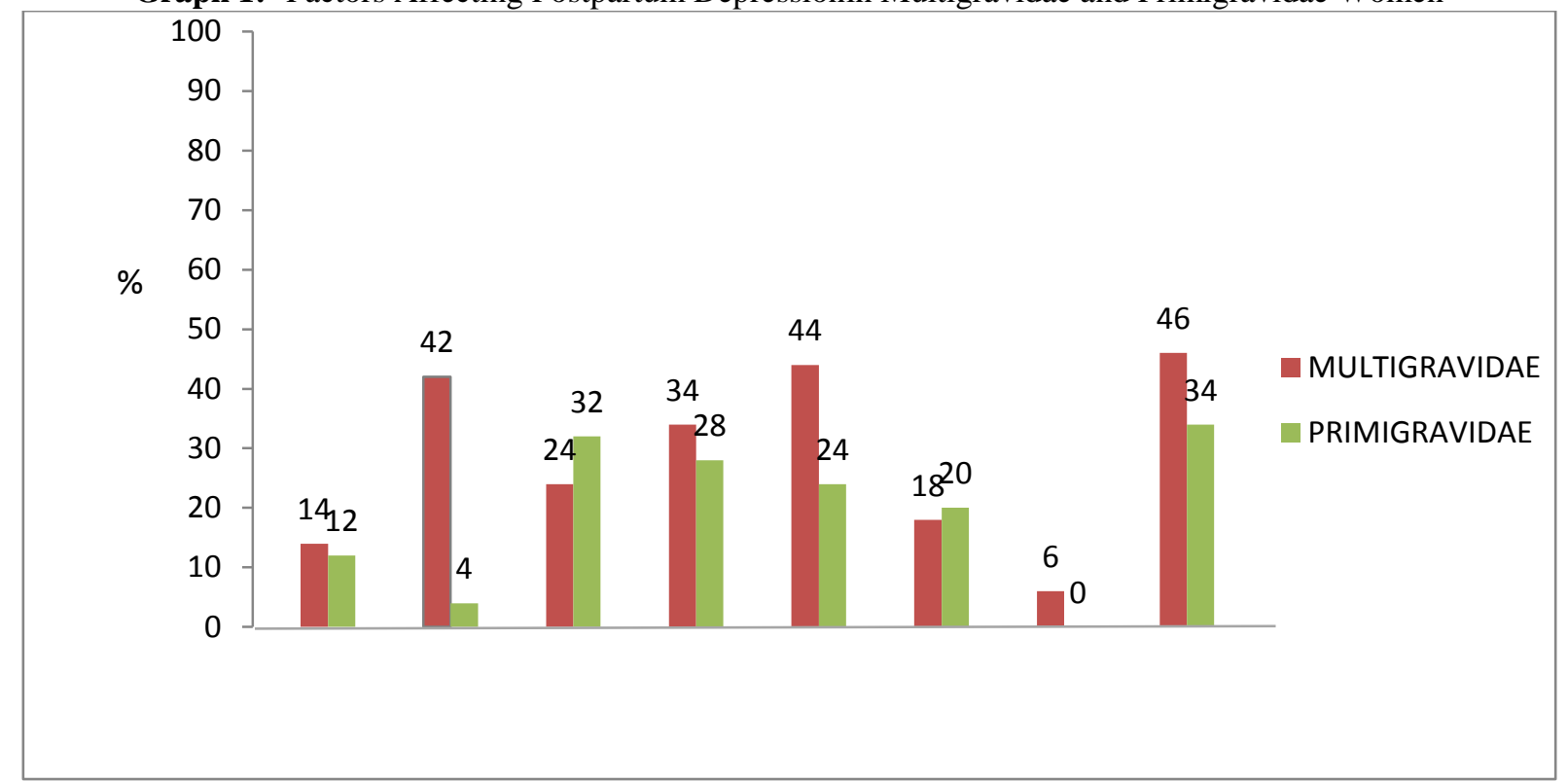

*A - Unwanted/Unplanned Pregnancy(Yes), * B-History Of Miscarriage(Yes) , *C- Lack Of Rest After Childbirth(Yes), *D -Lack Of Knowledge Regarding Childcare(Yes), *E - Feeling Tensed/Depressed During Pregnancy(Yes), *F -Stressful Life Events(Yes), *G- Family Supporrt(No), *H - Domestic Help(No)

\begin{tabular}{|l|c|c|}
\hline FACTORS AFFECTING POSTNATAL DEPRESSION & $\begin{array}{c}\text { MULTIGRAVIDAE } \\
(\%)\end{array}$ & $\begin{array}{c}\text { PRIMIGRAVIDAE } \\
(\%)\end{array}$ \\
\hline A-Unwanted/Unplanned Pregnancy(Yes) & 14 & 12 \\
\hline B-History Of Miscarriage(Yes) & 42 & 4 \\
\hline C-Lack Of Rest After Childbirth(Yes) & 24 & 28 \\
\hline D-Lack Of Knowledge Regarding Childcare(Yes) & 34 & 24 \\
\hline E-Feeling Tensed/Depressed During Pregnancy(Yes) & 44 & 20 \\
\hline F-Stressful Life Events(Yes) & 18 & 0 \\
\hline G-Family Supporrt(No) & 6 & 34 \\
\hline H-Domestic Help(No) & 46 & \\
\hline
\end{tabular}


Graph 2:- Levels Of EDPS Score in Multigravidae and Primigravidae Women

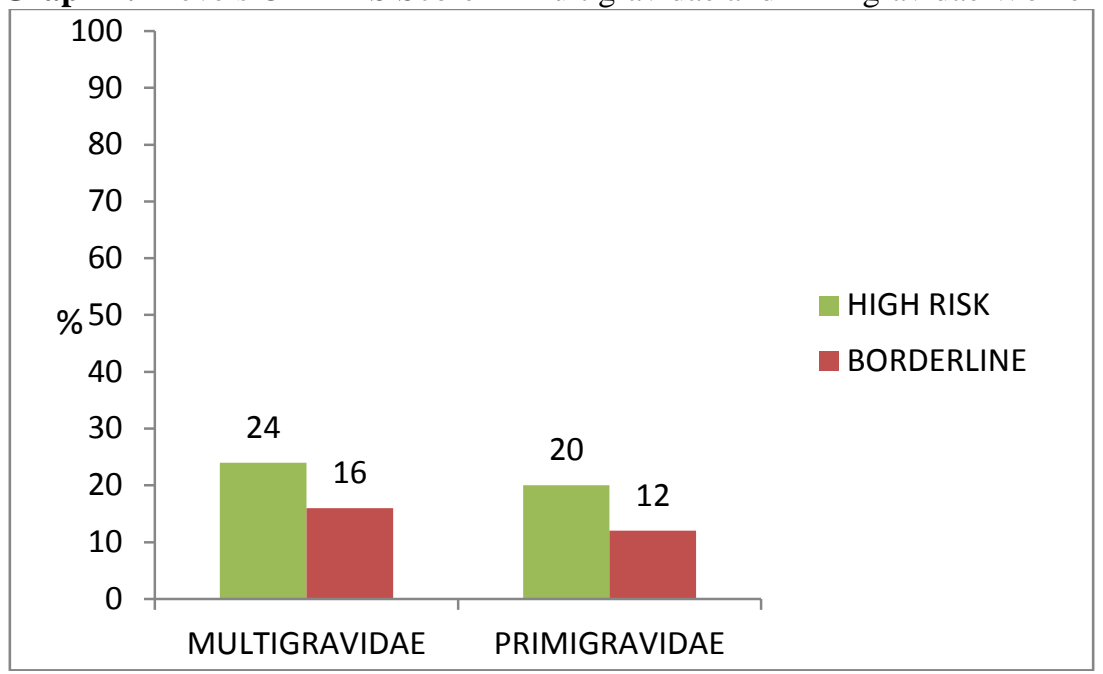

\begin{tabular}{|l|c|c|}
\hline EDPS & MULTIGRAVIDAE(N=50) & PRIMIGRAVIDAE(N=50) \\
\hline High Risk & 24 & 20 \\
\hline Borderline & 16 & 12 \\
\hline
\end{tabular}

Graph 3:- Comparison Between EDPS Scores of Primigravidae and Multigravidae Women

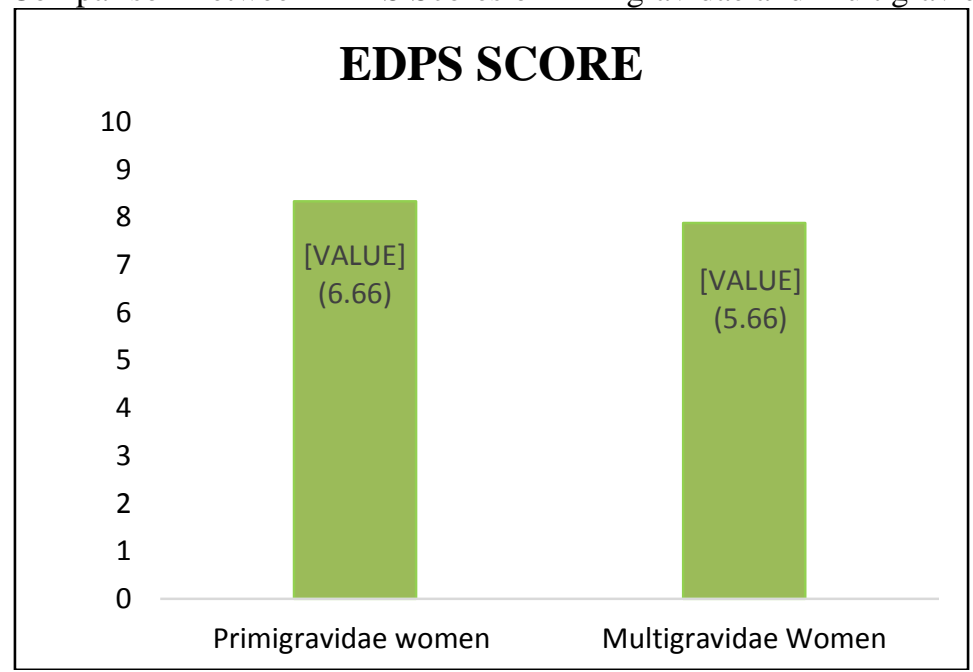

\begin{tabular}{|c|c|c|}
\hline \multicolumn{2}{|c|}{ Between Group Comparison } \\
\hline Groups & Mean \pm SD & P value \\
\hline Primigravidae Women $(\mathrm{N}=50)$ & $8.34 \pm 6.66$ & \multirow{2}{*}{0.983} \\
\hline Multigravidae Women $(\mathrm{N}=50)$ & $7.88 \pm 5.66$ & \\
\hline
\end{tabular}

\section{Results:-}

A total of 100 subjects (50 multigravidae ,50 primigravidae) were recruited for the study from Mumbai, navi Mumbai hospitals and maternity clinics. The mean age of the primigravidae \& multigravidae women were $24.54 \pm 3.8$ and $29.34 \pm 4.23$ years respectively. The mean age of marriage was $22.34 \pm 3.1$ years in primigravidae \& $22.5 \pm 5$ years in multigravidae women. Out of all the 100 subjects $80 \%$ women belonged to urban domicile and $20 \%$ to rural domicile. Out of 50 subjects of each group, socio economic status of 38\% Multigravidae \& $50 \%$ 
Primigravidae was good,42 \% Multigravidae women \& 42\%Primigravidae women was fair ,20\%Multigravidae \& $8 \%$ Primigravidae women was poor.Most of the Multigravidae women were gravida 2, 56\% Multigravidae \& $58 \%$ Primigravidae women delivered Male child, 48\% Multigravidae \& 42\% Primigravidae women delivered Female child. Graph 1 shows various factors responsible for developing postnatal depression. 14\% Multigravidae \& $12 \%$ Primigravidae women reported about unwanted/unplanned pregnancy. 42\% Multigravidae \& 4\%Primigravidae women reported about history of miscarriage.24\% Multigravidae \& 32\% Primigravidae women had lack of rest after childbirth. 34\% Multigravidae \& 28\% Primigravidae women had lack of knowledge regarding childcare.44\% Multigravidae \& 24\% Primigravidae women were feeling tensed/depressed during pregnancy.18\% Multigravidae \& $20 \%$ Primigravidae women had experienced stressful life events during pregnancy/post delivery i.e. still birth,painful delivery,family issues etc. 54\% Multigravidae \& 66\%Primigravidae women had domestic help. 6\% Multigravide women reported about lack of family support whereas none of the Primigravidae women had lack of family support.As shown in Graph 2 out of 100 women, 36\% in multigravidae women showed presence of postnatal depression.in which $24 \%$ multigravida \& $20 \%$ primigravida women had EDPS score falling in high risk category whereas $16 \%$ multigravidae \& 12\% Primigravidae women had EDPS score falling in borderline category. Graph 3 shows no statistical significant difference in between group comparison of primigravidae and multigravidae EDPS score.

\section{Discussion:-}

Postpartum Depression is characterized by depressive symptoms and a diagnosis of depression that occur several weeks after childbirth. The signs and symptoms of PPD are generally not distinguishable from major depressive disorders i.e. Postpartum blues, postpartum psychosis etc. that occurs at other times than postpartum period. But uncertain or indicisive feeling towards quality of motherhood are generally experienced with postpartum depression. A total of 100 subjects (50 multigravidae ,50 primigravidae) were recruited for the study from Mumbai, navi Mumbai hospitals and maternity clinics . $68 \%$ of women were housewives and $32 \%$ were working womens.Majority of the population had graduate level education. None of the women reported about any type of addiction. Around $26 \%$ husbands of these women had addictions such as alcohol, tobacco, cigarette etc.

The presence of postnatal depression was found to be $36 \%$ i.e. $20 \%$ multigravidae \& $16 \%$ primigravidae women. Out of 50 subjects from each group,unwanted or unplanned pregnancy(14\% multigravidae \& $12 \%$ primigravidae) , lack of rest after childbirth (24\% multigravidae \& $32 \%$ primigravidae), lack of knowledge regarding childcare(34\% multigravidae \& $28 \%$ primigravidae), ,experiencing stressful life events during pregnancy or after childbirth(18\% multigravidae \& $20 \%$ primigravidae), lack of domestic help(46\% multigravidae \& $34 \%$ primigravida) were found to be some of the contributing factors for postnatal depression in present study. In literature it has been seen that lack of family support is a contributing factor for postnatal depression [13] but in our study we found that, lack of family support was not a contributing factor of postnatal depression amongst primigravidae women whereas $6 \%$ multigravidae women reported that they don't have family support.Our results are also similar with the results obtained by Raid \& Meadows-oliver, where they reviewed 12 research articles examining postpartum depression in adolescent mothers and reported that family conflict, fewer social support, and low self-esteem were significant risk factors[13][14].

We also found that some of the stressful life events during pregnancy/post delivery which has strongly impacted them. Those were death of loved ones, excessive pain,still birth,health issues after conceiving, Previous history of miscarriage as reported by $42 \%$ multigravidae \& $41 \%$ primigravidae, feeling tensed/depressed during pregnancy reported (44\% multigravidae \& $24 \%$ primigravidae). Study done by Dr.Ashish \& Dr.Amit for screening of postnatal depression,statistical correlation was found between postpartum depression \& various associated risk factors i.e history of miscarriage,gender preference,feeling tensed/depressed during pregnancy etc.[15] These finding also supports the result of our study and can be attributed as associated risk factors for developing PPD.

The most common healthcare seeking barriers for lack of medical assistance were unawareness and the social pressures associated with the postpartum depression. Most of the women in our society are not aware about the altered mental state they can face in post-partum period and the condition is worsened when their health care provider also fail to teach them. A particular percentage of the women actually fail to identify and express their condition and avoiding any further medical assistance is also an important reason it is therefore mandatory to provide basic awareness regarding postpartum depression and instill the need to consult a medical advice in order to prevent the complications that is not only detrimental for one's lifestyle but also adversely affects a child's cognitive and emotional abilities. [16] 


\section{Conclusion:-}

Based on the results obtained in the present study it has been observed that about $16 \%$ primigravidae \& $20 \%$ multigravidae women showed presence of postpartum depression.We conclude that both the primigravidae as well as multrigravidae women showed the presence of post natal depression with EDPS score showing both high risk as well as borderline depression.

\section{Limitations and Further Scope Of Study}

Limitations of this study were smaller sample size thus reducing the generalization, language barrier, assessment of only hospital bound cases and one time data collection was done. Future study can be done on larger sample size and data can be collected at multiple intervals after the delivery.

\section{Referrences:-}

1. Ramsha Rukh,Huda Kafeel et.al.Prevalence Of Postpartum Depression in Primigravidae \& Multigravidae women with Normal Physiological Status,Faculty of Pharmacy, Jinnah University for women, KarachiPakistan.year

2. N Shrestha, P Hazrah, et.al. Incidence \& Prevalence of Postpartum Depression in a rural Community of India Journal of Chitwan Medical College 2015; 5(12): 11-19.

3. Dr. Ashish V Gokhale, Dr. Amit Vaja, Screening for Postpartum Depression, GUJARAT MEDICAL JOURNAL / DECEMBER - 2013 Vol. 68 No. 2.

4. Piyanee Klainin,David Gordon Arthur, Postpartum depression in Asian cultures: A literature review , Singapore. Alice Lee Centre for Nursing Studies, National University of Singapore, International Journal of Nursing Studies 46 (2009): 1355-1373.

5. K.I. Mohammad, J. Gamble et.al. Prevalence and factors associated with the development of antenatal and postnatal depression among Jordanian women. Midwifery Journal. December 2011; 27(6 ) .e238-e245.

6. R.J.S. SAVARIMUTHU, P. EZHILARASU, et.al.Postpartum Depression in the community : a qualitative study from rural South India ,International Journal of Social Psychiatry. 2010. Vol 56(1): 94-102.

7. Dennis, C. L. Psychosocial and psychological interventions for prevention of postnatal depression: systematic review. BMJ, July 2005. 331(7507):15.

8. Cox J.L.; Holden J.M.; Sagovsky R. Detection of postnatal depression: development of the 10-item Edinburgh Postnatal Depression Scale. Br J Psychiatry. 1987.150: 782-6.

9. (9) Righette-Veltema M., Conne-Perreard E., et.al.Postpartum depression and mother-infant relationships at 3 months old. Journal of Affective Disorders. 2002.70 (3):291-306.

10. American Psychiatric Association, 2000. Diagnostic and Statistical Manual of Mental Disorders (4th ed.; text revision). American Psychiatric Press, Washington, DC.

11. World Health Organization. International Statistical Classification of Diseases and Related Health Problems 10th Revision (ICD-10).Retrieved on April 4 , 2007 from http://www.who.int/classifications/apps/icd/icd10online/.

12. Field T. Postpartum depression effects on early interactions, parenting, and safety practices: A review. Infant Behavior and Development.Feb2010; 33( 1):1-6.

13. Elizabeth A. Howell, Pablo Mora, Howard Leventhal,Correlates of Early Postpartum Depressive Symptoms. Maternal child health journal.2006,March10(2):149-57.

14. Chen H., Chan Y.H. I||,et.al. Depressive symptomatology in pregnancy: Singaporean perspective. Social Psychiatry and Psychiatric Epidemiology.December2004.39 (12):975-979.

15. Raid V., Meadows-Oliver M., . Postpartum depression in adolescent mothers: an integrative review of the literature. Journal of Paediatric Health Care. September 2007. 21 (5):289-298.

16. Matijasevich A. et.al. Validation of Edinburgh Postnatal Depression Scale for screening of major depressive episode among adults from general population.BMC Psychiatry. October 2014;14:284. 